\title{
Dural Arteriovenous Malformations: A Review of the Literature and a Presentation of the JHN Series
}

\author{
Ed Marchan \\ Stavropoula Tjoumakaris \\ Robert H. Rosenwasser MD \\ Thomas Jefferson University \\ L. Fernando Gonzalez \\ Ciro Randazzo MD \\ Thomas Jefferson University
}

See next page for additional authors

Follow this and additional works at: https://jdc.jefferson.edu/jhnj

\section{Let us know how access to this document benefits you}

\section{Recommended Citation}

Marchan, Ed; Tjoumakaris, Stavropoula; Rosenwasser MD, Robert H.; Gonzalez, L. Fernando; Randazzo MD, Ciro; and Jabbour, Pascal MD (2010) "Dural Arteriovenous Malformations: A Review of the Literature and a Presentation of the JHN Series," JHN Journal: Vol. 5 : Iss. 2 , Article 2.

DOI: https://doi.org/10.29046/JHNJ.005.2.002

Available at: https://jdc.jefferson.edu/jhnj/vol5/iss2/2

This Article is brought to you for free and open access by the Jefferson Digital Commons. The Jefferson Digital Commons is a service of Thomas Jefferson University's Center for Teaching and Learning (CTL). The Commons is a showcase for Jefferson books and journals, peer-reviewed scholarly publications, unique historical collections from the University archives, and teaching tools. The Jefferson Digital Commons allows researchers and interested readers anywhere in the world to learn about and keep up to date with Jefferson scholarship. This article has been accepted for inclusion in JHN Journal by an authorized administrator of the Jefferson Digital Commons. For more information, please contact: JeffersonDigitalCommons@jefferson.edu. 
Dural Arteriovenous Malformations: A Review of the Literature and a Presentation of the JHN Series

\section{Authors}

Ed Marchan, Stavropoula Tjoumakaris, Robert H. Rosenwasser MD, L. Fernando Gonzalez, Ciro Randazzo MD, and Pascal Jabbour MD 


\section{Dural Arteriovenous Malformations: A Review of the Literature and a Presentation of the JHN Series}

\author{
Edward M. Marchan, MD, Stavropoula I. Tjoumakaris, MD, \\ Robert H. Rosenwasser, MD, L Fernando Gonzalez, MD, \\ Ciro Randazzo, MD, MPH, Pascal M. Jabbour, MD \\ Department of Neurological Surgery, Thomas Jefferson University, Philadelphia, PA
}

\section{Risk Stratification and Management Strategies \\ Introduction}

\section{Epidemiology}

Dural arteriovenous malformations (DAVMs), also known as dural arteriovenous fistulas, are arteriovenous shunts from a dural arterial supply to a dural venous channel, typically supplied by pachymeningeal arteries and located near a major venous sinus. ${ }^{1}$ The etiology of these lesions is not fully understood. DAVMs in the pediatric population are associated with structural venous abnormalities, ${ }^{2}$ but the majority of DAVMs are thought to be acquired. Different etiologies have been implicated in this phenomenon, namely: sinus thrombosis, trauma or surgery. ${ }^{2-6}$

DAVMs have been reported in all age groups, but mainly in the fifth and sixth decades of life. ${ }^{7-9}$ The estimated incidence of DAVMs is 0.17 per $100,000^{10,11}$ and they are one fifth as common as arteriovenous malformations (AVMs). ${ }^{10,12}$ They represent $10 \%$ to $15 \%$ of all intracranial vascular malformations, ${ }^{10,12-17}$ with a higher incidence in women. In fact, a female/male ratio of $2 / 1$ exists in certain anatomic sites such as the cavernous and transverse-sigmoid sinuses. ${ }^{6-8,18-20} \mathrm{AVMs}$ are usually solitary, nonetheless in $5 \%$ of cases multiple lesions have been described. ${ }^{10,21,22}$

\section{Natural History}

DAVMs are usually acquired. ${ }^{6,23}$ Recent experimental work suggests that the wide array of clinical associations of DAVM may be explained by the development of venous obstruction and hypertension with aberrant angiogenesis. ${ }^{24}$ It is hypothesized that their development arises from altered angiogenesis within the dura following an inciting event such as trauma, surgery or chronicinfection. Sinusthromboses can be an accompanying factor in these cases. Cases have been documented of angiographically proven dural sinus thrombosis in which DAVMs subsequently developed as a consequence of the obstructed sinus. Next, initial microshunts proliferate in association with venous hypertension, and mature into clinically significant arteriovenous fistulae. The degree of progression or involution determines the significance of the abnormality. These fistulas may result in hemorrhage or other focal manifestations including hemodynamic insufficiency. DAVMs cause decreased regional cerebral blood flow in cortical regions where there is retrograde venous drainage. ${ }^{25}$

The development of DAVMs following trauma and surgery is well known. ${ }^{25,26}$ They have also been reported in association with chronic infection, vascular disease and tumors. ${ }^{27}$ Other cases of DAVM have no clear association. They may be identified at anatomical sites distinct from the presumed inciting event. The exact mechanism of development remains unclear. It is well established that the development of a DAVM in these diverse settings requires a common mechanism as well as a possible anatomic or genetic predisposition. ${ }^{28,29}$

An established DAVM may follow one of several unpredictable natural courses. Some lesions remain asymptomatic or maintain stable clinical symptomatology and angiographic features over many years. Others undergo spontaneous regression, involution and resolution with stabilization or improvement of neurological symptoms. ${ }^{30-35}$ Features that may predispose to such spontaneous involution are not known. In fact, DAVMs in the region of the cavernous sinus are particularly prone to this phenomenon with as many as 40 percent of reported cases having undergone spontaneous involution.
In contrast, some DAVMs may demonstrate increase in size from either arterial or venous enlargement ${ }^{1,12,36}$ or even de novo development. ${ }^{37}$ Pachymeningeal arterial feeders may be progressively recruited with enlargement of the nidus. The mechanisms behind this progressive recruitment of arterial feeders from numerous sources have not been elucidated. This phenomenon results in hypertrophy of dural arteries and the reappearance of involuted embryonic arteries that may not normally be visible in the adult dura mater. In some DAVMs there is also progression of pathology on the venous side. Progressive arterialization of the pathologic dural leaflets results in hypertension in adjacent leptomeningeal venous channels. This may lead to retrograde leptomeningeal venous drainage. Under arterialized pressures these channels may become tortuous and, eventually, varicose or aneurysmal. The catastrophic consequence that ensues is a cerebral hemorrhage from cortical venous drainage. In DAVMs that present with intracranial hemorrhage and have retrograde cortical venous drainage, there is a $35 \%$ risk of rebleeding within the first 2 weeks. ${ }^{38}$

\section{Presentation}

Clinical manifestations of DAVMs are highly variable and are related primarily to the location of the fistula as well as from the retrograde cortical venous drainage as described above. These range from minor symptoms to intracranial hemorrhage. The vast majority of symptoms can be attributed to the primary or secondary venous manifestations of the DAVM. Presentation may be sudden or slowly progressive. The degree and type of symptoms are determined by venous topography, venous flow pattern and the capacity of surrounding compensatory venous drainage. As above, the most serious neurological sequelae from DAVMs are associated with retrograde leptomeningeal venous drainage. ${ }^{12}$ Focal neurological deficits likely result from venous hypertension and intracranial hemorrhage from rupture of arterialized leptomeningeal veins. 
Table I: Classification of DAVMs

\begin{tabular}{|c|c|c|c|}
\hline Type & Djindjian & Cognard & Borden \\
\hline I & Normal antegrade flow into dural sinus & Normal antegrade flow into dural sinus & $\begin{array}{l}\text { Drains directly into venous sinus or menin- } \\
\text { geal vein }\end{array}$ \\
\hline \multirow[t]{4}{*}{$\|$} & \multirow[t]{4}{*}{$\begin{array}{l}\text { Drainage into venous sinus with reflux } \\
\text { into adjacent sinus or cortical vein }\end{array}$} & a. Retrograde flow into sinus & \multirow{4}{*}{$\begin{array}{l}\text { Drains into dural sinuses or pachymenin- } \\
\text { geal veins with retrograde drainage into } \\
\text { subarachnoid veins. }\end{array}$} \\
\hline & & b. Retrograde filling of cortical veins only & \\
\hline & & c. Retrograde drainage into sinus and cortical veins & \\
\hline & & $\begin{array}{l}\text { d. Drains into dural sinus or meningeal veins with } \\
\text { retrograde drainage into subarachnoid veins. }\end{array}$ & \\
\hline III & $\begin{array}{l}\text { Drainage into cortical veins with } \\
\text { retrograde flow }\end{array}$ & $\begin{array}{l}\text { Direct drainage into cortical veins with retro- } \\
\text { grade flow }\end{array}$ & $\begin{array}{l}\text { Drains into subarachnoid veins without } \\
\text { dural sinus or meningeal involvement }\end{array}$ \\
\hline IV & Drainage into venous pouch (lake) & $\begin{array}{l}\text { Direct drainage into cortical veins with venous } \\
\text { ectasia }>5 \mathrm{~mm} \text { and } 3 \mathrm{x} \text { larger than diameter of } \\
\text { draining vein }\end{array}$ & \\
\hline V & & Drainage to spinal perimedullary veins & \\
\hline
\end{tabular}

There are a wide variety of non-hemorrhagic symptoms. ${ }^{39,40}$ More benign symptoms of pain, tinnitus or bruit are related to arteriovenous shunting and flow in the DAVM. Pulsatile tinnitus or other subjective auditory symptoms may occur with or without pain. These symptoms are likely related to high flow through dural vascular channels at the base of the skull. Other painful complaints may be related to orbital congestion or stretching of dural leaflets by engorged vascular channels, or to direct compression of the trigeminal nerve by arterialized venous structures near the petrous apex. A variety of neuro-ophthalmologic manifestations of DAVMs include visual and gaze abnormalities caused by venous hypertension, as well as orbital or ocular venous hypertension with resulting orbital crowding, venous stasis retinopathy and glaucoma. Other cranial DAVMs may present with symptoms of increased intracranial pressure (ICP) or a poorly defined headache. ${ }^{41,42}$ While the headaches are non-specific, there does appear to be an association with the dysplastic changes in meningeal vessels which are often present in DAVMs . There are also a wide spectrum of presenting focal neurological symptoms including seizure, hearing loss, cranial nerve palsy, papilledema and other visual symptoms and focal motor/sensory deficits. ${ }^{43-47}$

DAVMs may also result in altered cerebrospinal (CSF) hydrodynamics. ${ }^{48}$ Dilated venous structures may act as mass lesions, obstructing the CSF circulation and causing hydrocephalus. In other cases, dural venous hypertension may result in decreased absorption of CSF with secondary intracranial hypertension and papilledema. This latter complication appears to be more common in association with high flow lesions draining into large dural venous sinuses and in the setting of concomitant dural sinus outflow obstruction.

Particular clinical presentations take place with DAVMs in specific locations. ${ }^{12,36,39,40}$ DAVMs in the region of the transverse or sigmoid sinus, or near the cavernous sinus, often drain into the associated venous sinuses and may cause a variety of clinical manifestations due to flow or local venous engorgement. A high flow in the region of the transverse-sigmoid sinus junction, for example, often results in pulsatile tinnutus, headache and bruit. This phenomenon does not lead to bleeding or cause other deficits unless there is associated retrograde leptomeningeal venous drainage. Lesions at the anterior cranial fossa or the tentorial incisura rarely drain into a patent dural venous sinus and are more frequently associated with leptomeningeal venous drainage. They are more likely to cause serious clinical sequelae from venous hypertension and hemorrhage. It is important to mention that hemorrhage has not been reported in the absence of this feature; in all published cases with carefully documented diagnostic studies, hemorrhage from DAVMs is associated with rupture of arterialized venous structures. The prognosis of a first hemorrhage from DAVMs is ominous, and is associated with a greater than 30 percent mortality or serious disability. Hemorrhage from DAVMs in anticoagulated patients has uniformly been fatal.

\section{Classification}

\section{Location}

Classification of DAVMs has evolved over time to be useful in guiding therapeutic intervention. Initial attempts were simplistic, emphasizing the anatomic location (e.g., transverse-sigmoid DAVM, cavernous DAVM, sagittal sinus DAVM, convexity DAVM, tentorial and posterior fossa DAVM). These lacked meaningful information in regard to predicting the nature or outcome of the abnormality or treatment options. Subsequent systems incorporated information from diagnostic angiography. ${ }^{5,49,50}$

\section{Venous drainage}

Perhaps one of the most well recognized classification schemes specific to DAVMs is that developed by Djindjian et al. ${ }^{49}$ This system categorizes a lesion into one of four types: type I DAVMs are characterized by normal anterograde drainage into a venous sinus or meningeal vein; type II lesions drain into a sinus, with reflux into adjacent sinuses or cortical veins; type III DAVMs drain directly into cortical veins with resultant retrograde flow into the cerebral venous compartment; type IV DAVMs have drainage directly into a venous pouch (venous lake or venous ectasia). They concluded from their study that type I DAVMs were benign, with each sequential type having more aggressive characteristics. Since the introduction of the Djindjian classification of DAVMs, other studies have been published in the literature attempting to correlate certain features of the DAVM with the likelihood of associated hemorrhage or other specific neurologic complications. ${ }^{12,39,51,52}$ 
With the advent of more effective endovascular therapeutic techniques, a means of predicting lesion risk and management options emerged. Cognard et al. developed a classification system derived from a modified version of that published by Djindjian et al. ${ }^{50}$ Cognard defined five types of DAVMs that are exclusively based on the pattern of venous outflow. As such, type I DAVMs were characterized by normal antegrade flow into the affected dural sinus. Type II lesions were associated with an abnormal direction of venous drainage within the affected dural sinus. These lesions could be further categorized into three subtypes: type IIa, lesions with retrograde flow exclusively into the sinus or sinuses; type IIb, lesions with retrograde venous drainage into the cortical veins only; and type II $a+b$, lesions with retrograde drainage into sinuses and cortical veins. Type III DAVMs drained directly into a cortical vein or veins without venous ectasia, whereas type IV DAVMs had drainage into cortical veins with the critical component of venous ectasia greater than $5 \mathrm{~mm}$ in diameter and three times larger than the diameter of the draining vein. A DAVM was considered to be type $\mathrm{V}$ when drainage was into spinal perimedullary veins. Correlation with their clinical data yielded the following conclusions: Type I DAVMs are considered benign, and treatment is usually not necessary, except possibly for palliation of symptoms; type IIa lesions are best treated with arterial embolization; while type IIb and IIa $+\mathrm{b}$ lesions usually require both transarterial and transvenous embolization for effective obliteration. For those lesions of types III through $\mathrm{V}$, transarterial and occasionally transvenous embolization aimed at complete occlusion of the fistula is necessary and usually will need to be combined with surgical techniques in eradicating the threatening cortical venous drainage.

Meanwhile, Borden, et al., also proposed a classification system emphasizing venous anatomy. ${ }^{5}$ This system is appealing in its simplicity, with only 3 categories. Type I dural AVMs drain directly into dural venous sinuses or pachymeningeal veins. Type II malformations drain into dural sinuses or pachymeningeal veins but also have retrograde drainage into subarachnoid (leptomeningeal) veins. Type III malformations drain solely into subarachnoid (leptomeningeal) veins and do not have dural sinus or meningeal venous drainage. The validity of both the Cognard and Borden classification systems were confirmed in 102 intracranial DAVMs in 98 patients. ${ }^{52}$ (Table I)

\section{Risk stratification}

Regardless of lesion location or previous clinical presentation or other symptomatology, the most important factor determining the propensity of a lesion to an aggressive clinical course appears to be the presence of leptomeningeal venous drainage. Lesions that drain into a large patent venous sinus may have various clinical associations but do not bleed or cause focal neurological deficits unless associated with retrograde leptomeningeal venous drainage. Lesions without drainage into a patent dural venous sinus more frequently are associated with leptomeningeal venous drainage and more often are prone to more serious clinical sequelae, such as an intracerebral hemorrhage. As discussed, the risk of hemorrhage appears to be related directly to the presence of tortuous and aneurysmal leptomeningeal arterialized veins in association with DAVMs.

\section{Treatment}

\section{General principles of treatment}

A DAVM may rarely be discovered on routine imaging studies on digital subtraction angiography performed for other indications. Incidental lesions must be carefully assessed for features predisposing to aggressive clinical behavior. Complete angiographic evaluation is indicated in every case of suspected DAVM unless the patient is a poor candidate for therapeutic intervention or refuses invasive diagnostic studies. Lesions should be evaluated specifically for the presence of leptomeningeal venous drainage and for any variceal, aneurysmal changes in the venous circulation or venous ectasia. In the absence of these features, the lesion should be followed expectantly. There is no evidence to justify prophylactic treatment of DAVMs that are not associated with leptomeningeal cortical venous drainage. Expectant follow-up of these lesions should include serial MR imaging for any evidence of development of leptomeningeal venous dilations. Angiographic re-examination of the lesion every few years should be considered, especially for DAVMs at the anterior cranial fossa or the tentorial incisura, which as discussed, very commonly harbor leptomeningeal venous drainage.

Definitive prophylactic treatment should be strongly considered for asymptomatic and incidentally discovered DAVMs with leptomeningeal venous drainage. The patient should be given the option of open surgical intervention or such alternative radiosurgical or endovascular options as may be appropriate for the specific lesion type and location. If treatment does not succeed at totally eliminating lepto- meningeal venous drainage, either further definitive therapy or very close follow-up of the lesion is indicated. It is our belief that anticoagulation is contraindicated in the setting of DAVMs with leptomeningeal venous drainage.

Definitive intervention for DAVMs that in the past behaved aggressively warrants more serious thought. Nevertheless, the morbidity of a first hemorrhage with DAVMs is substantial, and many patients do not survive or do not recover to a condition suitable for therapeutic intervention. Furthermore, little is known about the risk either of subsequent hemorrhage or of the progression of neurological deficits in this clinical setting. However, there are numerous documented cases of progression of focal neurological symptoms resulting in death or major disability unless the DAVM is obliterated. It is our recommendation that lesions that have hemorrhaged or that cause focal neurological symptoms due to parenchymal venous hypotension be considered for definitive treatment. Palliative therapy is not sufficient in this setting.

On the other hand, lesions that present with pain or pulsatile tinnitus are evaluated and treated in the same way as incidental lesions. Nonspecific measures aimed at the symptoms are usually sufficient. Palliative treatment of the DAVM may be considered for the control of symptomatology. Rarely is definitive treatment indicated solely for pain or pulsatile tinnitus. We do not believe that the risk of definitive treatment is justified in such DAVMs as they do not exhibit leptomeningeal venous drainage.

Lesions associated with ophthalmoplegia are evaluated on a case-by-case basis. Frequently, painful ophthalmoplegia will resolve spontaneously, and many such lesions involute after angiography. In other cases, ophthalmoplegia may be progressive or associated with retinopathy and visual loss. In such situations, treatment of the associated DAVM is justified. Palliative treatment may be sufficient to stabilize visual symptoms. Again, a radical cure of the DAVM should not be pursued at any risk, and is generally not warranted unless the symptoms are truly debilitating or the DAVM is associated with leptomeningeal venous drainage.

The management of DAVMs associated with papilledema and increased intracranial pressure has been discussed previously. Palliation or definitive cure of the DAVM frequently (but not always) results in reversal of papilledema and stabilization of visual symptoms. Again, in the absence of leptomeningeal venous drainage, the risk of radical lesion treatment 

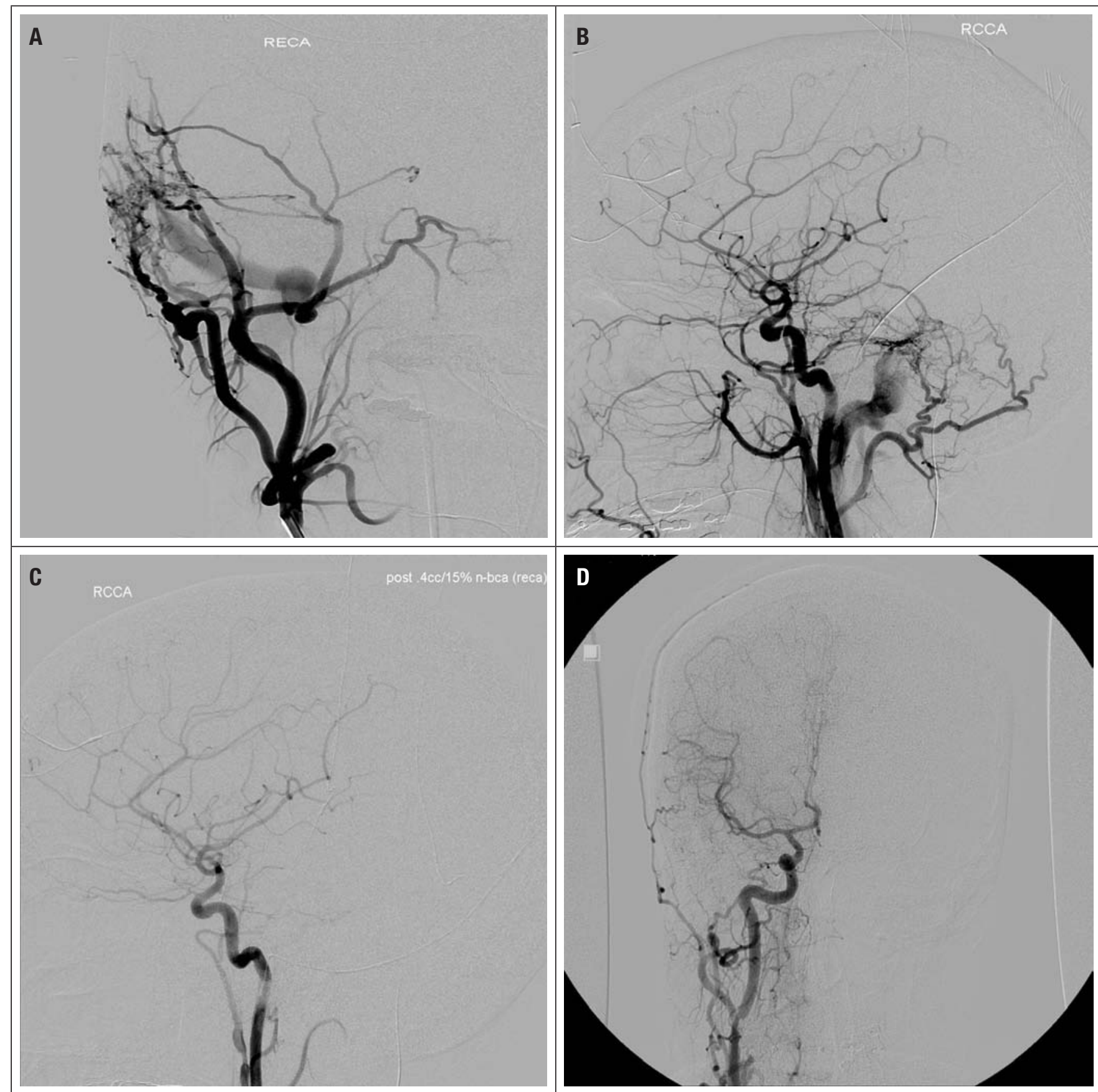

Figure 1

This is a 46 y/o female who underwent embolization of a Right Temporo-Occipital DAVM. A) and B) Pre-embolization digital substraction angiography (DSA) images showing multiple arterial feeders (mostly from the occipital-meningeal system). C) and D) Post-embolization DSA showing no supply from the meningeal system or occipital system after only one embolization session.

may not be justified, and may or may not result in subsequent control of intracranial hypertension. Cerebrospinal fluid diversion via lumboperitoneal shunting or optic nerve sheath decompression may be combined with transarterial embolization and or radiosurgery in the management of these entities. Transvenous occlusion is rarely possible in this setting, as it may further compromise intracranial venous outflow. Lumboperitoneal shunting or optic nerve sheath decompression may effectively treat 

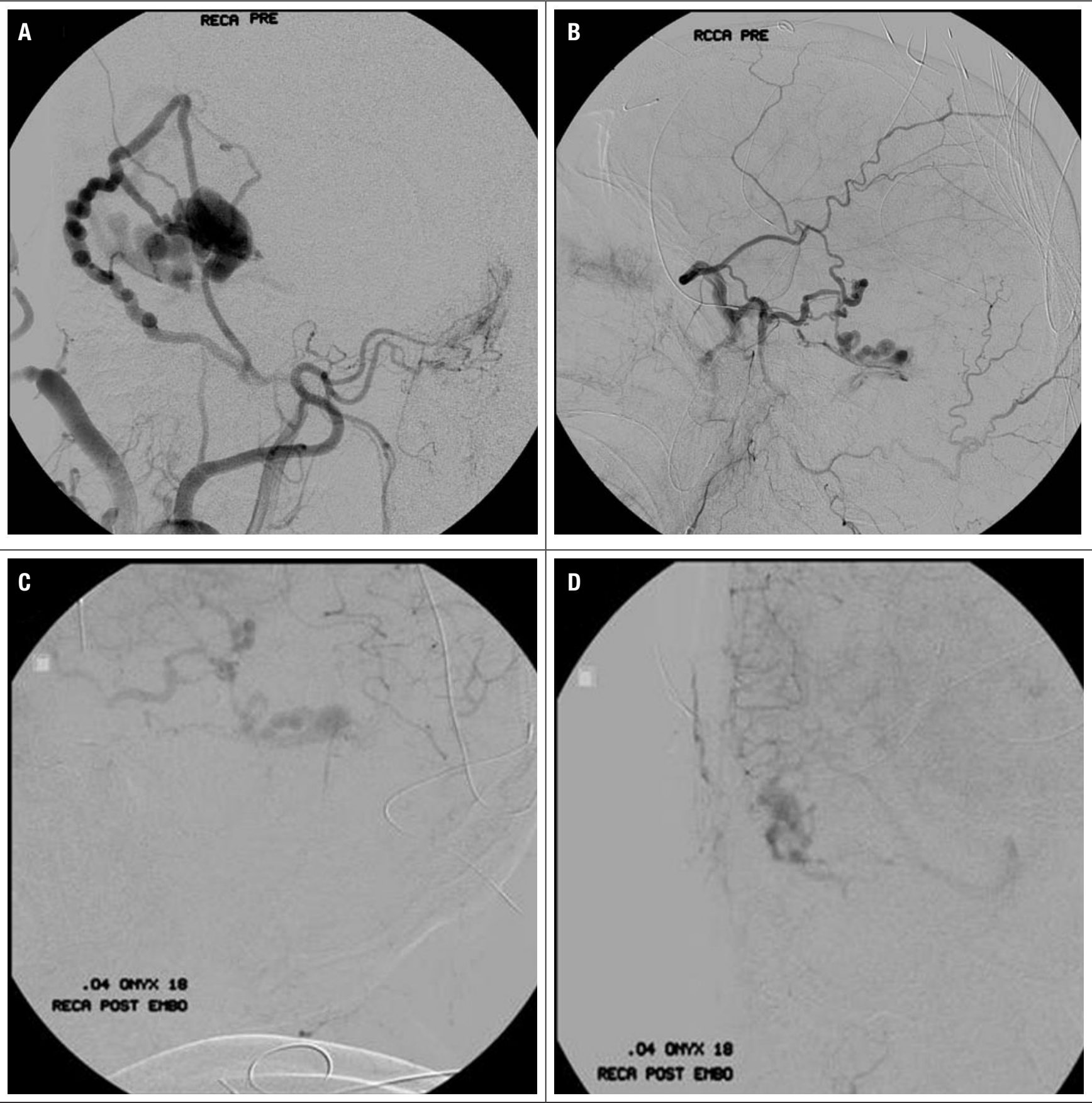

Figure 2

This is a 51 y/o male receiving two different embolizations for right-side DAVM. A) and B) Pre-embo DSA showing multiple ECA feeders (mostly from the occipital-meningeal system) and cortical venous drainage (CVD). C) and D) DSA after 2 different embolizations showing persistent fistula filling and CVD. This patient subsequently needed a craniotomy for eradication of CVD.

the secondary complications of papilledema while the DAVM is followed expectantly, treated palliatively, or with radiosurgery. Ventriculoperitoneal shunting may not be possible in view of small cerebral ventricles, and may be dangerous in the setting of arterialized cortical or subependymal veins.
In summary, clinical symptoms other than hemorrhage and progressive neurological deficits rarely warrant radical treatment of a DAVM, unless the lesion is particularly accessible 
or is associated with features predisposing to subsequent aggressive clinical behavior. Patient reassurance, symptomatic treatment, or lesion palliation is frequently sufficient. In DAVMs with features predisposing to an aggressive clinical course, a more definitive treatment strategy should be adopted. It is obvious that the myriad of clinical manifestations of DAVMs and the wide spectrum of possible angiographic and pathophysiologic scenarios call for highly individualized management strategies. Diagnostic investigation should be thorough so as to identify DAVMs with features predisposing to aggressive clinical behavior such as leptomeningeal cortical venous drainage or venous ecstasia. Treatment strategies should include a highly individualized choice of modalities from the available armamentarium of symptomatic treatment, lesion palliation, transarterial and /or transvenous endovascular therapy, open surgical invention, and radiosurgery. For the foreseeable future, the treatment of DAVMs should preferably be entrusted to multidisciplinary teams with expertise in the recognition and management of these lesions, and with experience in a variety of treatment options approaches.

\section{Treatment strategy and location Surgery}

The goals of surgical treatment of DAVMs include 1) physical interruption or obliteration of arterialized leptomeningeal venous connections and 2) maximal coagulation or excision of the pathological dura. There is a continuous risk for significant blood loss. This is particularly true early in the procedure when incomplete exposure may be accompanied by significant bleeding. From the early steps of skin infiltration and incision, the operation should proceed in small steps, with hemostatic control before the subsequent step. This approach is indeed more speedy, efficient and safe than having to take time to control brisk bleeding from many sources. As a rule, no incision should be made unless one was prepared to control catastrophic bleeding from it. A thorough review of preoperative angiography and judicious use of preoperative embolization helps to limit this risk. Continuous communication with the anesthesia team is critical.

Meticulous attention to hemostasis and microsurgical technique is imperative throughout the procedure. ${ }^{53}$ Following identification, resection of the abnormal dura is aided by the irrigating bipolar. Small permanent vascular clips in tandem, alternated with dural transection may be useful. Temporary aneurysm clips are helpful in decision making prior to coagulation and sectioning of variceal veins, which may significantly impact adjacent cortical venous circulation. It is sometimes possible to identify discrete arteriovenous connections whose occlusion significantly decreases surrounding subarachnoid venous engorgement.

Direct puncture of large varices with intraoperative placement of obliteratingcoils has been used successfully. ${ }^{54}$ A combination of coils and glue after obtaining access by craniectomy and direct sinus puncture has also been reported. ${ }^{55}$ Resection of the dural sinus can be accomplished without the risk of venous infarction if the resected segment is arterialized and collateral channels are well developed. ${ }^{56}$ In some cases, surgical clipping of the draining vein close to the DAVM, with extensive dural coagulation rather than resection may be preferred. ${ }^{57}$ Recently, presigmoid skull base exposures have been employed, specifically for access to petrosal and sigmoid lesions. ${ }^{58}$ Image guided frameless navigation is useful for flap design and localization of DAVMs or associated cortical venous drainage. Intraoperative angiography helps to ensure complete resection in difficult cases.

\section{Endovascular techniques}

Transarterial embolization has been widely used in the treatment of DAVMs. ${ }^{1,59,60}$ The use of flow-guided catheter technology and increased experience with particle and glue embolization as well as detachable coils have greatly improved the safety and efficacy of this method. ${ }^{61-63}$ However, transarterial embolization rarely succeeds in totally eliminating and curing a DAVM, except in rare instances of limited fistulae with a small number of accessible feeders. More commonly, DAVMs involve a multitude of feeders, which often arise as small twigs from major cerebral arteries that are not amenable to embolization. While transarterial embolization may obliterate the filling of the lesion after one injection, the DAVM often continues to draw feeders from other sources, and will reappear on subsequent angiography, possibly in a more ominous configuration. DAVMs that are partially treated with transarterial embolization may later recur and progress to catastrophic hemorrhage.

What transarterial embolization can do is to palliate disabling symptoms even when it does not totally cure the DAVM. Symptom palliation may be accomplished by transarterial embolization of external carotid artery feeders to the DAVM, although such an intervention is not without risk and rarely succeeds in totally eliminating the DAVM. Arterial embolization may give a false sense of security that the lesion was "treated" while the DAVM may progress to acquire more aggressive features including leptomeningeal venous drainage (even in the absence of recurrent symptoms). DAVMs that are followed expectantly or treated palliatively should be monitored closely with serial diagnostic studies to watch for the development of leptomeningeal venous drainage, which may occur without change in clinical symptoms. Such lesions should continue to be followed as discussed above. Transarterial embolization also plays an important role in decreasing flow through DAVMs prior to surgical intervention, transvenous obliteration, or radiosurgery. ${ }^{64-66}$ This adjunctive, preparatory use of transarterial embolization has greatly enhanced the safety and efficacy of other more definitive treatment measures.

Noninvasive imaging methods, including MRI and MRA may be used for interval studies, although these modalities may miss subtle development of leptomeningeal venous drainage, which may be clinically catastrophic. Depending on the clinical situation and the particular lesion, serial MR studies may be performed on a yearly basis, with formal angiography every few years or sooner if symptoms change, or if there is a suggestion of new leptomeningeal venous drainage on MRI .

Transvenous endovascular obliteration of DAVMs has recently been used with good results. ${ }^{67-69}$ This modality aims at the thrombosis of the venous side of the lesion, often including the obliteration of the adjacent dural venous sinus. Occlusion of the venous side of DAVMs is usually well tolerated if the pathologic dural sinus is arterialized and does not serve as a site of drainage of cerebrovenous circulation. Instead, the pathologic dural segment is often associated with harmful retrograde leptomeningeal venous drainage, and these channels are secondarily obliterated with thrombosis of the venous side of DAVMs. This strategy has been used most successfully for the treatment of DAVMs with accessible venous drainage. Transvenous obliteration is particularly effective in the treatment of cavernous sinus DAVMs (access through the inferior petrosal sinus), although these lesions frequently do not require any therapeutic intervention because of their benign clinical symptomatology and tendency toward spontaneous regression. 
Transvenous obliteration has also been used in cases of transverse-sigmoid sinus DAVMs, and may be substantially safer than open surgical approaches to these lesions. However, there may be no accessible transvenous route for many DAVMs, including tentorial incisura DAVMs and anterior cranial fossa DAVMs, which frequently behave aggressively. Transvenous obliteration may occasionally be performed after open surgical exposure, through puncture of the dural venous sinus or the arterialized venous varix and the injection of coils or glue. ${ }^{54}$ Rarely, transvenous occlusion may result in propagating venous thrombosis or altered hemodynamic patterns with paradoxical clinical deterioration or hemorrhage. Occasionally, a DAVM will recur adjacent to endovascularly occluded venous sinus, and this could represent reconstitution of arteriovenous channels within the walls of the occluded sinus, or in the organized thrombus within the sinus channel. These cases are amenable to surgical excision of the segment of occluded sinus, and disconnection of associated arterialized leptomeningeal veins.

\section{Radiosurgery}

The goal of radiosurgical treatment is sclerosis and obliteration of arteriovenous connections within the pathological dura, resulting in secondary thrombosis of the DAVM. Advantages include non-invasive treatment and avoidance of risks associated with invasive procedures. Disadvantages include delayed response to treatment and risk of radiation injury to normal structures in the vicinity of the DAVM. When combined with transarterial embolization, $95 \%$ of patients demonstrated symptomatic improvement and $87 \%$ demonstrated angiographic cure on angiography performed a median of 12 months following radiosurgery. ${ }^{70}$ There was an acceptable complication rate with this treatment strategy. Radiosurgery alone was effective when the DAVM was not amenable to embolization but the time course for symptomatic improvement was longer.

DAVMs of the transverse-sigmoid sinuses treated with a similar strategy yielded a $96 \%$ symptom resolution or significant improvement and a total or near-total obliteration at mean angiography 21 months following radiosurgery. ${ }^{66}$ There were no intracerebral hemorrhages or radiation-related complications. While the ideal treatment parameters and ultimate role of radiosurgery continue to evolve, it has an established role in the multimodality treatment of DAVMs.

\section{The Thomas Jefferson Experience}

\section{Background}

We have amassed the longest reported followup of 39 patients who were initially treated by endovascular means. These patients were subsequently evaluated throughout their treatment plan in order to discern whether multi-modality therapy would be necessary or not or whether strict endovascular therapy would suffice. We hereby report their outcomes and complications. Our follow-up involves patients who were electively referred to our institution who had a non-invasive study (such as an MRA) illustrating the DAVM as well as those who presented with an intracerebral hemorrhage. The sequence of events involved a diagnostic angiogram followed by endovascular embolization as the primary means of treatment.

Thirty-nine patients (22 males and 17 females) underwent endovascular treatment of dural AVMs at our primary institution from 20012009. Ages ranged from 39-71 (mean age: 48). Seventy-nine percent of patients had cortical venous drainage.

\section{Methods}

We studied the following outcome factors: 1) the number of embolizations needed to obliterate the lesion, and 2) the percentage of patients requiring transvenous embolization, craniotomy, or radiosurgery for eradication of the lesion after endovascular management.

We also inspected whether those patients who were initially found to have retrograde cortical venous drainage had subsequent eradication of this phenomenon after multi-modality therapy. Finally, we assessed post operative complications from either of the modalities.

\section{Results}

\section{Outcomes toward Obliteration}

\section{of Fistulous Component}

The number of endovascular embolizations performed in order to decide on whether to proceed with surgery, radiosurgery or ascertain complete obliteration of the fistulas was 2.1. This was an average obtained by adding up the number of endovascular embolizations needed to obtain at least a 95\% reduction in fistula flow in all patients studied. We deemed the DAVM as "treated" when this result was obtained as per the observation of the angiographer. Seventyone percent $(71 \%)(28 / 39)$ of patients had complete obliteration of the fistula; 21 of those by endovascular means and 7 via craniotomy. Figure 1 illustrates eradication of the DAVM after one embolization session.
Of the eleven patients who did not have complete obliteration of the fistula, seven of those (64\%) had at least $90 \%$ obliteration with only one feeding pedicle remaining. Three patients needed radiosurgery as a final approach for treatment. The average dose utilized for eradication of the DAVM was $22 \mathrm{~Gy}$ in single fraction.

Outcomes Toward Eradication of Retrograde Cortical Venous Drainage

We studied whether retrograde cortical venous drainage would be eradicated by various components of multi-modality therapy. $87 \%$ $(26 / 30)$ of patients had full obliteration of their cortical venous drainage. Of these 26 patients, $69 \%(18 / 26)$ had obliteration obtained by endovascular means while the rest needed surgical venous ligation. Figure 2 illustrates treatment of a patient by multi-modality therapy to eradicate both the cortical venous drainage phenomenon as well as the fistulous component. It is interesting to note that in five of these 18 patients who had CVD treated endovascularly, success was only obtained when a transvenous approach was performed.

\section{Complications}

The following complications were noticed: epidural infection after craniotomy (1), post op intracranial hemorrhage not requiring surgery after embolization (1), and need for vein patch procedure after uncontrolled femoral bleeding (1). All above complications were diagnosed and treated expeditiously.

\section{Analysis}

Endovascular treatment of DAVMs is a safe and effective way to treat these complex lesions. Additional modalities such as surgery and radiosurgery have become adjuvant in the treatment of these lesions. We strongly believe based on this data that endovascular embolization should be the primary modality for treating DAVMs

\section{Prognosis and outcome}

An established DAVM has an unpredictable natural course, varying from spontaneous regression to more venous recruitment and hemorrhage, depending on the presence or absence of leptomeningeal venous drainage. The prognosis of a first hemorrhage from DAVMs is ominous, and has been shown to be associated with greater than $30 \%$ mortality or serious disability. The annual bleeding risk of a DAVM varies between 1.8 to $15 \%,{ }^{71}$ the risk of rebleed over the period of two weeks has been estimated to be around $35 \% .{ }^{38}$ The acute or gradual progression from one grade to the next has been described. 
Any change in the bruit or symptoms of a DAVM should warrant reevaluation to rule out the development of alternative drainage paths such as leptomeningeal drainage pathways. Recently carotid duplex sonography has been reported as a tool for follow up of treated dural AVMs, with a correlation of the resistance index of the external carotid artery with the effectivness of treatment. ${ }^{72}$

\section{Conclusion}

Much has been learned in recent years about the pathoanatomy, pathophysiology, natural history and therapeutic options for DAVMs. A better understanding of these lesions has allowed more prompt and precise diagnosis, and a realistic assessment of features predisposing to aggressive clinical course. Treatment is not only guided toward the palliation of clinical symptoms, but as importantly, toward prevention of future sequelae. The therapeutic armamentarium includes a number of options with varying risk and effectiveness for individual lesions. Transarterial embolization, transvenous embolization, surgical therapy or radiosurgery can be used alone or in various combinations as required for individual clinical scenarios.

\section{References}

1. Lasjaunias, P., et al., Radiological anatomy of the vascularization of cranial dural arteriovenous malformations. Anat Clin 1984. 6(2): p. 87-99.

2. Jafar, J., I. Awad, and P. Huang, Intracranial vascular malformations: Clinical decisions and multimodality management strategies, in Vascular malformations of the central nervous system, J. Jafar, I. Awad, and R. Rosenwasser, Editors. 1999, Lippincott Williams \& Wilkins: Philadelphia. p. 219-232.

3. Awad, I., The diagnosis and management of intracranial dural arteriovenous malformations. Contemp Neurosurg, 1991. 13: p. 1-6.

4. Awad, I., Dural arteriovenous malformations., in Neurovascular surgery., L. Carter and R. Spetzler, Editors. 1994, McGraw-Hill: New York. p. 905-932.

5. Borden, J.A., J.K. Wu, and W.A. Shucart, A proposed classification for spinal and cranial dural arteriovenous fistulous malformations and implications for treatment. J Neurosurg, 1995. 82(2): p. 166-79.

6. Houser, O.W., et al., Arteriovenous malformation affecting the transverse dural venous sinus-an acquired lesion. Mayo Clin Proc, 1979. 54(10): p. 651-61.

7. Chaloupka, J., C. Putman, and T. Roth, Diagnostic evaluation, in Techniques in Neurosurgery. 1996, Lippincott-Raven: Philadelphia. p. 5-25.

8. Fermand, M., et al., Long term follow-up of 43 pure dural arteriovenous fistulae (AVF) of the lateral sinus. Neuroradiology, 1987. 29(4): p. 348-53.

9. Obrador, S., M. Soto, and J. Silvela, Clinical syndromes of arteriovenous malformations of the transverse-sigmoid sinus. J Neurol Neurosurg Psychiatry, 1975. 38(5): p. 436-51.

10. Flemming, K. and R. Brown, Natural history of intracranial vascular malformations., in Neurological surgery., R. Winn, Editor. 2004, Elsevier.: Philadelphia. p. 2159-2183.

11. Brown, R.D., Jr., et al., Frequency of intracranial hemor rhage as a presenting symptom and subtype analysis: a population-based study of intracranial vascular malformations in Olmsted Country, Minnesota. J Neurosurg, 1996. 85(1): p. 29-32
12. Awad, I.A., et al., Intracranial dural arteriovenous malformations: factors predisposing to an aggressive neurological course. J Neurosurg, 1990. 72(6): p. 839-50.

13. Luessenhop, A., Dural arteriovenous malformations., in Neurosurgery., R. Rengachary, Editor. 1986, McGraw-Hill.: New York. p. 1473-1477.

14. Grady, M.S. and L. Pobereskin, Arteriovenous malformations of the dura mater. Surg Neurol, 1987. 28(2): p. 135-40.

15. Aminoff, M.J., Vascular anomalies in the intracranial dura mater. Brain, 1973. 96(3): p. 601-12.

16. Brown, R.D., Jr., D.O. Wiebers, and D.A. Nichols, Intracranial dural arteriovenous fistulae: angiographic predictors of intracranial hemorrhage and clinical outcome in nonsurgical patients. J Neurosurg, 1994. 81(4): p. 531-8.

17. Malik, G.M., et al., Venous angiomas: an underestimated cause of intracranial hemorrhage. Surg Neurol, 1988. 30(5): p. $350-8$.

18. Houser, O.W., et al., Intracranial dural arteriovenous malformations. Radiology, 1972. 105(1): p. 55-64

19. Newton, T. and W. Hoyt, Dural arteriovenous shunts in the region of the cavernous sinus. Neuroradiology., 1970. 1: p. 71-81.

20. Toya, S., et al., Spontaneous carotid-cavernous fistula during pregnancy or in the postpartum stage. Report of two cases. $J$ Neurosurg, 1981. 54(2): p. 252-6.

21. Nakamura, M., et al., Two unusual cases of multiple dural arteriovenous fistulas. Neurosurgery, 1997. 41(1): p. 288-92; discussion 292-3.

22. Ushikoshi, S., Y. Kikuchi, and K. Miyasaka, Multiple dural arteriovenous shunts in a 5-year-old boy. AJNR Am J Neuroradiol, 1999. 20(4): p. 728-30.

23. Chaudhary, M.Y., et al., Dural arteriovenous malformation of the major venous sinuses: an acquired lesion. AJNR Am J Neuroradiol, 1982. 3(1): p. 13-9.

24. Lawton, M.T., R. Jacobowitz, and R.F. Spetzler, Redefined role of angiogenesis in the pathogenesis of dural arteriovenous malformations. J Neurosurg, 1997. 87(2): p. 267-74.

25. Ishikawa, T., et al., Development of anterior cranial fossa dural arteriovenous malformation following head trauma. Case report. J Neurosurg, 1997. 86(2): p. 291-3.

26. Nabors, M.W., et al., Delayed postoperative dural arteriovenous malformations. Report of two cases. J Neurosurg, 1987. 66(5): p. 768-72.

27. Yokota, M., et al., Meningioma in sigmoid sinus groove associated with dural arteriovenous malformation: case report. Neurosurgery, 1993. 33(2): p. 316-9; discussion 319.

28. Singh, V., et al., Dural arteriovenous fistula associated with prothrombin gene mutation. J Neuroimaging, 2001. 11(3): p. 319-21.

29. Yassari, R., B. Jahromi, and R. Macdonald, Dural arteriovenous fistula after craniotomy for pilocytic astrocytoma in a patient with protein S deficiency. Surg Neurol, 2002. 58(1): p. 59-64; discussion 64

30. Magidson, M.A. and P.E. Weinberg, Spontaneous closure of a dural arteriovenous malformation. Surg Neurol, 1976. 6(2): p. $107-10$.

31. Hansen, J.H. and I. Sogaard, Spontaneous regression of an extra- and intracranial arteriovenous malformation. Case report. J Neurosurg, 1976. 45(3): p. 338-41.

32. Luciani, A., et al., Spontaneous closure of dural arteriovenous fistulas: report of three cases and review of the literature. AJNR Am J Neuroradiol, 2001. 22(5): p. 992-6.

33. Kiyosue, H., et al., Treatment of intracranial dural arteriovenous fistulas: current strategies based on location and hemodynamics, and alternative techniques of transcatheter embolization. Radiographics, 2004. 24(6): p. 1637-53.

34. Bitoh, S. and S. Sakaki, Spontaneous cure of dural arteriovenous malformation in the posterior fossa. Surg Neurol, 1979. 12(2): p. 111-4.

35. Olutola, P.S., et al., Spontaneous regression of a dural arteriovenous malformation. Neurosurgery, 1983. 12(6): p. 687-90.
36. Awad, I.A. and D. Barrow, Dural arteriovenous malformations. 1993, Park Ridge, IL.: American association of neurological surgeons.

37. Friedman, J.A., B.E. Pollock, and D.A. Nichols, Development of a cerebral arteriovenous malformation documented in an adult by serial angiography. Case report. J Neurosurg, 2000. 93(6): p. 1058-61.

38. Duffau, H., et al., Early rebleeding from intracranial dural arteriovenous fistulas: report of 20 cases and review of the literature. J Neurosurg, 1999. 90(1): p. 78-84.

39. Lasjaunias, P., et al., Neurological manifestations of intracranial dural arteriovenous malformations. J Neurosurg, 1986. 64(5): p. 724-30.

40. Vinuela, F., et al., Unusual clinical manifestations of dural arteriovenous malformations. J Neurosurg, 1986. 64(4): p. 554-8.

41. Chimowitz, M.I., et al., Intracranial hypertension associated with unruptured cerebral arteriovenous malformations. Ann Neurol, 1990. 27(5): p. 474-9.

42. Cognard, C., et al., Dural arteriovenous fistulas as a cause of intracranial hypertension due to impairment of cranial venous outflow. J Neurol Neurosurg Psychiatry, 1998. 65(3): p. 308-16.

43. Kim, M.S., et al., Intraosseous dural arteriovenous fistula of the skull base associated with hearing loss. Case report. Neurosurg, 2002. 96(5): p. 952-5.

44. Rizzo, M., E.P. Bosch, and C.E. Gross, Trigeminal sensory neuropathy due to dural external carotid cavernous sinus fistula. Neurology, 1982. 32(1): p. 89-91.

45. Willinsky, R., et al., Tortuous, engorged pial veins in intracranial dural arteriovenous fistulas: correlations with presentation, location, and MR findings in 122 patients. AJNR Am Neuroradiol, 1999. 20(6): p. 1031-6.

46. Benndorf, G., et al., Tentorial dural arteriovenous fistula presenting with various visual symptoms related to anterior and posterior visual pathway dysfunction: case report. Neurosurgery, 2003. 53(1): p. 222-6; discussion 226-7.

47. Lee, P.H., et al., Parkinsonism as an initial manifestation of dural arteriovenous fistula. Eur J Neurol, 2005. 12(5): p. 403-6.

48. Gelwan, M.J., et al., Dural arteriovenous malformations and papilledema. Neurosurgery, 1988. 22(6 Pt 1): p. 1079-84.

49. Djindjian, R., J.J. Merland, and J. Theron, Superselective arteriography of the external carotid artery. 1977, New York.: Springer-verlag. 606-628.

50. Cognard, C., et al., Cerebral dural arteriovenous fistulas: clin ical and angiographic correlation with a revised classification of venous drainage. Radiology, 1995. 194(3): p. 671-80.

51. Malik, G.M., et al., Dural arteriovenous malformations and intracranial hemorrhage. Neurosurgery, 1984. 15(3): p. 332-9.

52. Davies, M.A., et al., The validity of classification for the clinical presentation of intracranial dural arteriovenous fistulas. $J$ Neurosurg, 1996. 85(5): p. 830-7.

53. Grisoli, F., et al., Surgical treatment of tentorial arteriovenou malformations draining into the subarachnoid space. Report of four cases. J Neurosurg, 1984. 60(5): p. 1059-66.

54. Endo, S., et al., Direct packing of the isolated sinus in patient with dural arteriovenous fistulas of the transverse-sigmoid sinus. J Neurosurg, 1998. 88(3): p. 449-56.

55. Houdart, E., et al., Transcranial approach for venous embolization of dural arteriovenous fistulas. J Neurosurg, 2002. 97(2): p. 280-6.

56. Sundt, T.M., Jr. and D.G. Piepgras, The surgical approach to arteriovenous malformations of the lateral and sigmoid dural sinuses. J Neurosurg, 1983. 59(1): p. 32-9.

57. Hoh, B.L., et al., Surgical management of high-grade intracranial dural arteriovenous fistulas: leptomeningeal venous disruption without nidus excision. Neurosurgery, 1998. 42(4): p. 796-804; discussion 804-5.

58. Kattner, K.A., T.C. Roth, and S.L. Giannotta, Cranial base approaches for the surgical treatment of aggressive posterior fossa dural arteriovenous fistulae with leptomeningeal drainage: report of four technical cases. Neurosurgery, 2002. 50(5): p. 1156-60; discussion 1160-1. 
59. Hardy, R.W., et al., External carotid cavernous fistula treated by transfemoral embolization. Surg Neurol, 1978. 9(4): p. 255-6.

60. Vinuela, F.V., et al., Detachable calibrated-leak balloon for superselective angiography and embolization of dural arteriovenous malformations. J Neurosurg, 1983. 58(6): p. 817-23.

61. Nesbit, G.M. and S.L. Barnwell, The use of electrolytically detachable coils in treating high-flow arteriovenous fistulas. AJNR Am J Neuroradiol, 1998. 19(8): p. 1565-9.

62. Jansen, O., et al., Endovascular therapy of arteriovenous fistulae with electrolytically detachable coils. Neuroradiology, 1999. 41(12): p. 951-7.

63. Liu, H.M., et al., Transarterial embolisation of complex cavernous sinus dural arteriovenous fistulae with low-concentration cyanoacrylate. Neuroradiology, 2000. 42(10): p. 766-70.
64. Goto, K., et al., Combining endovascular and neurosurgical treatments of high-risk dural arteriovenous fistulas in the lateral sinus and the confluence of the sinuses. J Neurosurg, 1999. 90(2): p. 289-99.

65. Collice, M., et al., Surgical treatment of intracranial dural arteriovenous fistulae: role of venous drainage. Neurosurgery, 2000. 47(1): p. 56-66; discussion 66-7.

66. Friedman, J.A., et al., Results of combined stereotactic radiosurgery and transarterial embolization for dural arteriovenous fistulas of the transverse and sigmoid sinuses. J Neurosurg, 2001. 94(6): p. 886-91.

67. Halbach, V.V., et al., Transvenous embolization of dural fistulas involving the cavernous sinus. AJNR Am J Neuroradiol, 1989. 10(2): p. 377-83.
68. Halbach, V.V., et al., Transvenous embolization of dural fistulas involving the transverse and sigmoid sinuses. AJNR Am J Neuroradiol, 1989. 10(2): p. 385-92.

69. Roy, D. and J. Raymond, The role of transvenous embolization in the treatment of intracranial dural arteriovenous fistulas. Neurosurgery, 1997. 40(6): p. 1133-41; discussion $1141-4$

70. Pollock, B.E., et al., Stereotactic radiosurgery and particulate embolization for cavernous sinus dural arteriovenous fistulae. Neurosurgery, 1999. 45(3): p. 459-66; discussion 466-7.

71. Steiger, H.J., D. Hanggi, and R. Schmid-Elsaesser, Cranial and spinal dural arteriovenous malformations and fistulas: an update. Acta Neurochir Suppl, 2005. 94: p. 115-22.

72. Tsai, L.K., et al., Carotid duplex sonography in the followup of intracranial dural arteriovenous fistulae. AJNR Am J Neuroradiol, 2005. 26(3): p. 625-9.

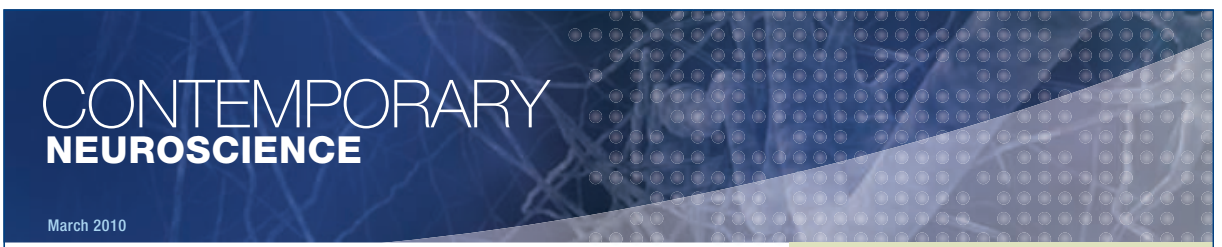

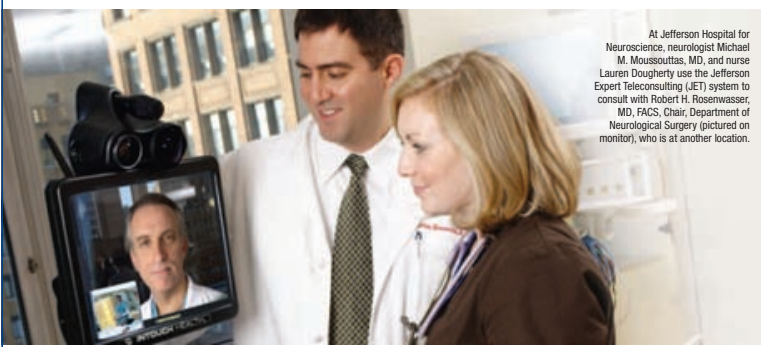

Announcing Jefferson's Teleconsulting (JET) Robotic System for Help with Diagnosis and Treatment of Time-Sensitive Neurovascular Diseases

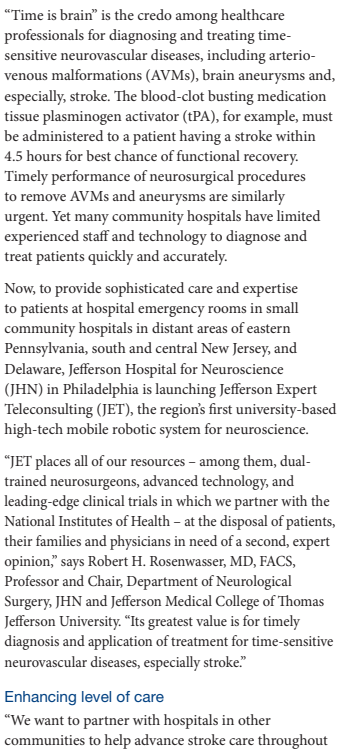

the region. As the leading provider of stroke care in the region, we have an obligation to do that," adds Pamela Kolb, Vice President, Neuroscience Service, $J \mathrm{HN}$.

Supported by Thomas Jefferson University, a leading academic center JHN is the region's only dedicated hospital for neuroscience and leading most experienced and comprehensive center for diagnosis and treatment of stroke and cerebrovascula disease. JHN's Acute Stroke Center is the largest such facility, with more board-certified neurocritical care physicians than most, in the greater Delaware Valley. It is also a Joint Commission-accredited primary stroke center.

"Stroke is the third-leading cause of death in the United States but the leading cause of disability, by a notes. "Given its prevalence and time-sensitive nature, it is alarming how few people receive treatment in the appropriate amount of time. With JET, even hospitals in remote areas can provide patients with expert consultation and disposition of appropriate care from an experienced neurologist or neurological surgeon immediately in cases where every minute can make critical difference. It's a very cost-effective approach providing 24/7 onsite coverage and expertise.

How JET works

Each participating hospital is supplied with a mobile robotic plattorm, manufactured by InTouch Health" hysicin to be remotely present JET's panoramic shich afford physicians, patients and hospital staff a safe and effective interactive experience.

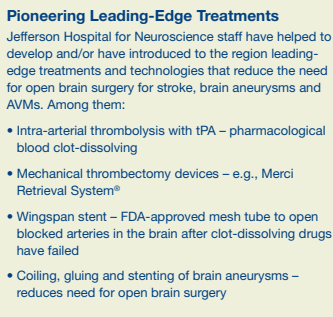

Coiling, gluing and stenting of brail
reduces need for open brain surgen

InTouch's remote presence devices are the first and only with FDA approval. The robots allow direct connection to medical devices such as electronic stethoscopes, otoscopes and ultrasound to transmit medical data to the remote physician.

"Should a patient arrive in the ER of a hospital that's located, say, three hours away from Jefferson and either doesn't have a neurologist or neurosurgeon with a neurosurgeon," Dr. Rosenwasser, explains, "the attending physician contacts JHN. The JHN Network specialist on call then uses a laptop to connect to the remote hospital via the robot, obtain a medical history by speaking directly with the patient and/or family members, examine the patient and determin what therapy is immediately needed, in real time, without delay. Mnally, a decision is made either to admit the patient to the local hospitals Critical Care

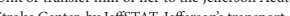
stric

"Patients may not need to be transferred - they can stay in their own community and be treated very successfully there, thanks to this program," says Ms Kolb. "And most pationts want to stay close to home JET enables them to receive sophisticated medica care withou having to travel long distances."

"It also serves as an educational program," adds Dr. Rosenwasser. "As a teaching center, JHN will use JET to share our clinical protocols with participating hospitals so that they can enhance stroke care withi their communities. On the other hand, should the patient need an advanced neurosurgical procedure
[See sidebar], we can arrange for prompt transfer" JHN continues to set the standard worldwide
for state-of-the-art care for time-sensitive
neurovascular diseases. For more information neurovascular diseases. For more information
about these and other treatments, visit

\section{Jefferson.}

oscience 